Portland State University

PDXScholar

Electrical and Computer Engineering Faculty

Publications and Presentations

$12-1-1977$

\title{
Light extinction in polydisperse particulate systems
}

Lee W. Casperson

Portland State University

Follow this and additional works at: https://pdxscholar.library.pdx.edu/ece_fac

Part of the Electrical and Computer Engineering Commons

Let us know how access to this document benefits you.

\section{Citation Details}

Lee W. Casperson, "Light extinction in polydisperse particulate systems," Appl. Opt. 16, 3183-3189 (1977).

This Article is brought to you for free and open access. It has been accepted for inclusion in Electrical and Computer Engineering Faculty Publications and Presentations by an authorized administrator of PDXScholar. Please contact us if we can make this document more accessible: pdxscholar@pdx.edu. 


\title{
Light extinction in polydisperse particulate systems
}

\author{
Lee W. Casperson
}

\begin{abstract}
Analytic methods are developed for determining the attenuation characteristics of light beams propagating through ensembles of scattering particles including those with complex index of refraction. The methods are applicable to a wide range of practical particulate systems, and the results are expressed explicitly in terms of elementary functions. Propagation through fog and clouds is considered as an example, and it is found that absorption by the liquid water in such ensembles affects strongly the extinction for ir wavelengths.
\end{abstract}

\section{Introduction}

In recent years light scattering methods have been employed in a wide variety of application areas. In one general class of applications scattering provides an important diagnostic technique for remote and nondestructive determination of the characteristics of discrete particles or general particulate systems. Examples range from simple smoke detection to pollution monitoring from spacecraft. Recently scattering techniques have been used in the analysis of submicroscopic viruses and other biological specimens. The main area of interest here is related to the general subject of optical communication. With optical information transmission it is essential to be able to estimate the losses that a specific particle distribution will introduce into a transmission link. Typical sources of attenuation include dust, smoke, haze, and fog. The purpose of this work is to develop analytical techniques for predicting the extinction characteristics of light beams propagating through systems of scattering particles.

In Sec. II various functions are discussed for describing the size distribution of the particles in the scattering ensemble. It is shown that a basic Poissonlike distribution is sufficient to characterize many of the particle systems encountered in practice. The advantages of this distribution become apparent in Sec. III, where explicit closed-form expressions are obtained for the extinction of the optical beams. The starting point for the scattering calculations is the anomalous diffraction method of van de Hulst. ${ }^{1}$ The formalism is generalized in Sec. IV to include absorbing particles, and propagation through fog is considered as an example.

The author is with University of California, School of Engineering \& Applied Science, Los Angeles, California 90024.

Received 25 February 1977.

\section{Particulate System Characterization}

Before any detailed scattering calculations can be performed, it is necessary to obtain a reasonable mathematical description of practical particle ensembles. Typically the index of refraction may be assumed to be constant from particle to particle for any specific ensemble. In the following paragraphs several size distributions are considered, and data-fitting procedures are described for use with these distributions.

The sizes of the particles in a system of scatterers can be represented in a variety of ways, and for some applications simple tabular or graphical representations are adequate. Often, however, it is extremely useful to be able to represent the size distribution in terms of an analytic function. Such a characterization can, for example, simplify greatly the calculation of the scattering or exinction of a light beam propagating through the system. In the past many different analytic distribution functions have been used to describe the particle ensembles encountered in practice. If the deviations in particle size are small compared to the dimensions of a typical particle, a simple Gaussian distribution is often adequate. However, many practical particle distributions do not satisfy this stringent narrowness criterion. In this case a difficulty with the Gaussian distribution is that it may imply a significant number of particles with negative dimensions. Therefore, it is highly desirable to employ skewed distribution functions which vanish for negative radii.

In the literature various types of two-parameter distributions have been employed. Occasionally more general three-parameter distributions have also been tried, but it is usually concluded that the quality of obtainable data does not warrant the more complex three parameter fit. ${ }^{2}$ One general class of functions includes the logarithmic distributions ${ }^{3}$

$$
p_{n}(a)=C_{n} a^{n} \exp \left[-\left(\ln a-\ln a_{n}\right)^{2} / 2 \sigma_{n}{ }^{2}\right]
$$

with the normalization constant 


$$
C_{n}{ }^{-1}=(2 \pi)^{1 / 2} \sigma_{n} a_{n}{ }^{n+1} \exp \left[(n+1)^{2} \sigma_{n}{ }^{2} / 2\right] .
$$

Equation (1) describes the distribution in radii of an ensemble of spherical particles with $a$ as the radial coordinate. For any particular value of $n$, the adjustable parameters are $a_{n}$ and $\sigma_{n}$. The functions given by Eq. (1) do have the desirable property of vanishing for $a=0$. The most popular special cases of Eq. (1) include the logarithmic normal distribution ${ }^{4}$

$$
p_{-1}(a)=C_{-1} a^{-1} \exp \left[-\left(\ln a-\ln a_{-1}\right)^{2} / 2 \sigma_{-1}{ }^{2}\right]
$$

and the zero order logarithmic distribution ${ }^{5}$

$$
\left.p_{o}(a)=C_{o} \exp \left[-\left(\ln a-\ln a_{o}\right)^{2} / 2{\sigma_{o}}^{2}\right)\right] .
$$

In order to make meaningful theoretical predictions about the scattering characteristics of a specific ensemble of particles, it is first necessary to fit the experimental data to one of the distributions implied by Eq. (1) or to some other equivalent function. Ideally this fitting should be accomplished by means of some type of least squares technique. However, satisfactory results can be obtained by a simpler procedure. First it is convenient to introduce the new dimensionless size parameter $y=a / a_{n}$, so that Eq. (1) reduces to

$$
p_{n}(y)=C_{n}\left(a_{n}\right)^{n+1} y^{n} \exp \left[-(\ln y)^{2} / 2 \sigma_{n}^{2}\right] .
$$

If Eq. (5) is renormalized to a peak value of unity, this new distribution becomes

$$
p_{n}{ }^{\prime}(y)=y^{n} \exp \left[-(\ln y)^{2} / 2 \sigma_{n}{ }^{2}-\left(n \sigma_{n}\right)^{2} / 2\right] .
$$

The two most fundamental and readily determined characteristics of practical size distribution data are the value of $a$ at which the distribution peaks and the width of the peak (typically measured at half-maximum). These two values may be used directly to determine the two parameters of the distribution. From Eq. (6) the distribution peaks at the normalized radius

$$
y_{\max }=\exp \left(n \sigma_{n}^{2}\right) \text {. }
$$

It also follows from Eq. (6) that the full width of the size distribution at half-maximum can be written

$$
\Delta y=2 y_{\max } \sinh \left[\sigma_{n}(2 \ln 2)^{1 / 2}\right] .
$$

The parameters which are assumed to be known from experimental data are $a_{\max }$ and $\Delta a$. But the ratio $\Delta a / a_{\max }$ is equal to the ratio $\Delta y / y_{\max }$, and Eq. (8) implies that one parameter of the fitted distribution is

$$
\sigma_{n}=(2 \ln 2)^{-1 / 2} \ln \left\{\left(\Delta y / 2 y_{\max }\right)+\left[\left(\Delta y / 2 y_{\max }\right)^{2}+1\right]^{1 / 2}\right\} .
$$

When $n$ has been specified the second parameter is given by $\mathrm{Eq}$. (7) in the form

$$
a_{n}=a_{\max } \exp \left(-n \sigma_{n}^{2}\right) .
$$

The next step of this discussion is to introduce an alternative family of two-parameter particle size distributions which for many scattering applications is much simpler to work with. This family involves the general class of power-law-exponential functions

$$
p(a)=C_{n} a^{n} \exp \left[-\left(a / a_{n}\right)^{m}\right]
$$

with the normalization constant

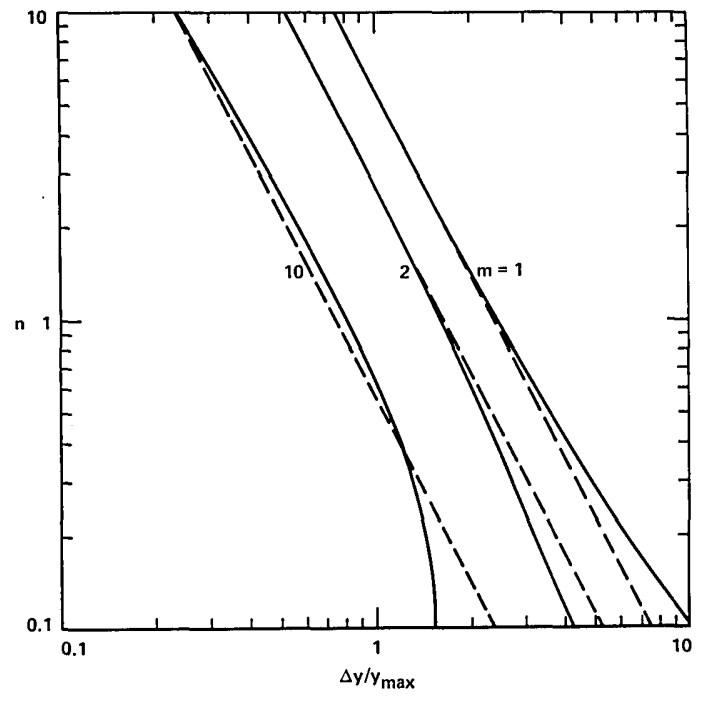

Fig. 1. Index $n$ of the power-law-exponential distribution functions vs the normalized width ratio $\Delta y / y_{\max }$ for various values of the parameter $m$. The dashed line in these plots represents the approximate formula $\Delta y / y_{\max }=(8 \ln 2 / \mathrm{mn})^{1 / 2}$.

$$
C_{n}^{-1}=\left(a_{n}\right)^{n+1} \Gamma[(n+1) / m] / m .
$$

When $m$ has been specified the coefficients to be determined are $n$ and $a_{n}$. If Eq. (11) is renormalized to a maximum value of unity, this distribution is

$$
p^{\prime}(y)=(m / n)^{n / m} y^{n} \exp \left(-y^{m}+n / m\right) .
$$

The maximum value occurs at the normalized radius

$$
y_{\max }=(n / m)^{1 / m} \text {. }
$$

The full width of the distribution given in Eq. (13) cannot be written explicitly for arbitrary values of $m$ and $n$. However, these results can be readily determined using Newton's method, ${ }^{6}$ and the results are plotted in Fig. 1. This is a graph of $n$ vs the ratio $\Delta y /$ $y_{\max }$ for various values of $m$. Thus, when $m$ has been specified, the value of the parameter $n$ follows from the experimentally determined ratio $\Delta y / y_{\max }$. The remaining parameter $a_{n}$ is given by Eq. (14) in the form

$$
a_{n}=a_{\max }(m / n)^{1 / m} \text {. }
$$

The dashed lines in Fig. 1 represent the approximate relationship

$$
\frac{\Delta y}{y_{\max }}=\left(\frac{8 \ln 2}{m n}\right)^{1 / 2}
$$

which is obtained from the Gaussian large $n$ limit of Eq. (13).

Two general families of particle distributions have now been considered, and techniques have been presented for fitting these distributions to an experimental data set. The next step is to determine which formula is to be preferred in the sense of providing the best fit to size distribution data encountered in practice. To make this evaluation it is helpful to consider some ex- 
amples graphically. In Fig. 2 is a plot of the functions implied by Eqs. (6) and (13) for the typical case $\Delta y / y_{\max }$ $=1$. In these graphs the logarithmic functions are all represented by the curve labeled $L$, whereas the various power-law-exponential functions are distinguished by the value of the index $m$. As noted previously all these curves vanish at $y=0(a=0)$. For completeness the corresponding Gaussian function is also shown, and this curve is labeled $G$.

The most significant feature of Fig. 2 is the close similarity between the plots of the various distributions. The quality of data obtained in determinations of particle sizes would usually not permit one to select one of the skewed distributions in preference to the others. Also, there is seldom any physical basis for supposing that one function might be more appropriate than another. The conclusion, therefore, is that the choice of an analytic size distribution function may often be made on the grounds of mathematical simplicity. Some of the functions shown in Fig. 2 are much easier to work with than others in succeeding scattering calculations. The preceding conclusion can be further underscored by considering somewhat narrower size distributions. Figure 3 is similar to Fig, 2 except that now the basic width ratio is $\Delta y / y_{\max }=0.4$. The agreement between the various distributions is even closer than before, and for clarity no attempt was made to represent the intermediate $m=1$ and $m=2$ curves.

\section{Extinction Coefficient}

In general the calculation of scattering from a dielectric sphere can involve some fairly formidable mathematical complexities. The exact Lorentz-Mie solutions are only obtained when computing facilities are available. For many applications approximate analytical solutions have proved to be highly useful. In the limit of small particles with refractive index close to unity, the most appropriate formalism is the Rayleigh-Debye approximation. With larger particles useful results are obtained from the anomalous diffraction method in which straight line propagation is assumed for the transmitted rays. ${ }^{1}$ The principal constraint on this method is that the index of refraction of the particle must be close to that of the host medium. It is, however, a remarkable fact that the anomalous diffraction results provide good agreement with most features of the exact solutions even when this condition is not well satisfied. Thus, the approximate extinction formulas are often adequate with relative indices of refraction as large as 1.5.7 Furthermore, these slight inaccuracies will often be outweighed by more fundamental uncertainties in the particle concentration, refraction, and size distribution. The anomalous diffraction method also loses some accuracy for values of $k a$ less than about $k a \sim 1$. However, the scattering efficiency is small for $k a \leqslant 1$, so that the total scattering may be dominated by the larger particles in the ensemble satisfying $k a \gtrsim 1$. This result will be discussed further in the following sections.

One basic result from anomalous diffraction theory is the extinction efficiency per particle $Q_{\text {ext }}$. The actual extinction cross section of a particle is equal to $Q_{\text {ext }}$

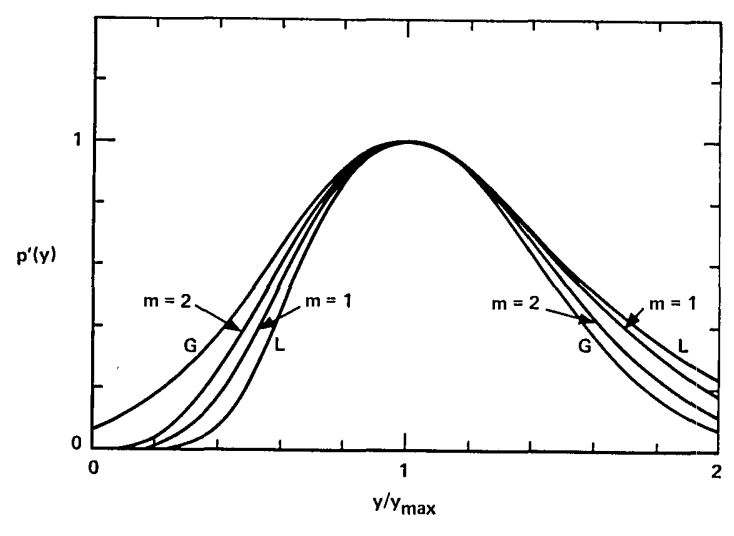

Fig. 2. Comparison of various size distributions including the logarithmic normal $(L)$, Gaussian $(G)$, and power-law-exponential functions $(m=1$ and $m=2)$. The normalized width ratio in these plots is $\Delta y / y_{\max }=1$.

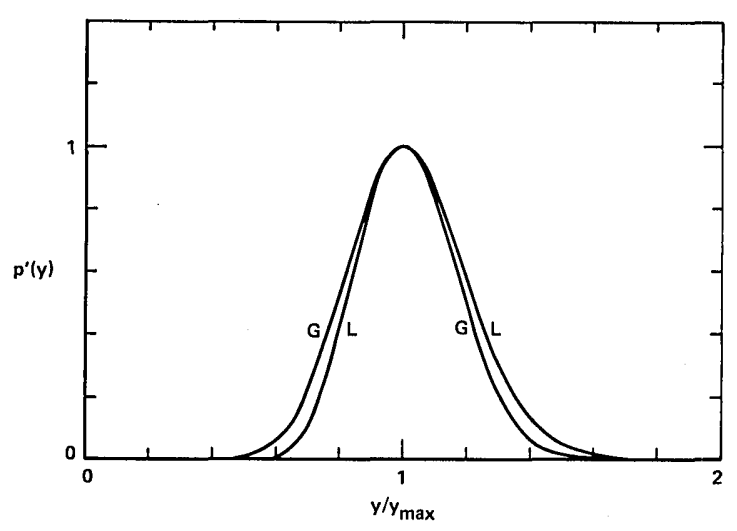

Fig. 3. Comparison of the logarithmic normal $(L)$ and Gaussian $(G)$ size distributions for a normalized width ratio of $\Delta y / y_{\max }=0.4$. The $m=1$ and $m=2$ power-law-exponential functions are intermediate between those shown and are omitted for clarity.

times the geometric cross-sectional area of the particle $\pi a^{2}$. The extinction efficiency is governed by the explicit equation

$$
Q_{\text {ext }}(\rho)=2-(4 / \rho) \sin \rho+\left(4 \dot{\rho}^{2}\right)(1-\cos \rho)
$$

where the dimensionless parameter $\rho$ is related to the index of refraction of the particle $\eta_{2}$, the index of refraction of the surrounding medium $\eta_{1}$, and the vacuum wavelength $\lambda$ by $\rho=4 \pi\left(\eta_{2}-\eta_{1}\right) a / \lambda$.

For an ensemble of scattering particles the exponential extinction coefficient can be written

$$
\gamma=\pi N_{o} \int_{0}^{\infty} a^{2} Q_{\mathrm{ext}}(a) p(a) d a,
$$

where $N_{o}$ is the concentration of scatterers and $p(a)$ is their size distribution. For purposes of this study the most convenient size distribution from Sec. II is the $m$ $=1$ power-law-exponential, which when normalized has the form 


$$
p(a)=\frac{1}{a_{n} \Gamma(n+1)}\left(\frac{a}{a_{n}}\right)^{n} \exp \left(-a / a_{n}\right) .
$$

With Eqs. (17) and (19) the integral in Eq. (18) can be written

$$
\begin{aligned}
\gamma=\frac{\pi a_{n}^{2} N_{o}}{\Gamma(n+1)} \int_{0}^{\infty}\{2 & -\frac{4}{\kappa y} \sin (\kappa y) \\
& \left.+\frac{4}{(\kappa y)^{2}}[1-\cos (\kappa y)]\right\} y^{n+2} \exp (-y) d y,
\end{aligned}
$$

where the coefficient $\kappa=4 \pi\left(\eta_{2}-\eta_{1}\right) a_{n} / \lambda$ has been introduced, and the dimensionless radius $y=a / a_{n}$ is again used.

The integrations indicated in Eq. (20) can be performed, and the result is ${ }^{8}$

$$
\begin{gathered}
\gamma=\frac{\pi a_{n}^{2} N_{o}}{\Gamma(n+1)}\left\{2 \Gamma(n+3)-\frac{4 \Gamma(n+2)}{\kappa\left(1+\kappa^{2}\right)^{(n+2) / 2}} \sin \left[(n+2) \tan ^{-1}(\kappa)\right]\right. \\
\left.+\frac{4 \Gamma(n+1)}{\kappa^{2}}-\frac{4 \Gamma(n+1)}{\kappa^{2}\left(1+\kappa^{2}\right)^{(n+1) / 2}} \cos \left[(n+1) \tan ^{-1}(\kappa)\right]\right\} .
\end{gathered}
$$

The gamma functions can be eliminated by means of the recurrence equation $\Gamma(z+1)=z \Gamma(z)$, and Eq. (21) reduces to

$$
\begin{aligned}
\gamma= & \frac{\pi a_{\max }^{2} N_{o}}{n^{2}}\left\{2(n+2)(n+1)-\frac{4(n+1)}{\kappa\left(1+\kappa^{2}\right)^{(n+2) / 2}} \sin [(n+2)\right. \\
& \left.\left.\times \tan ^{-1}(\kappa)\right]+\frac{4}{\kappa^{2}}-\frac{4}{\kappa^{2}\left(1+\kappa^{2}\right)^{(n+1) / 2}} \cos \left[(n+1) \tan ^{-1}(\kappa)\right]\right\} .
\end{aligned}
$$

Here the most likely particle radius $a_{\max }=a_{n} n$ has also been introduced.

Equation (22) is an explicit expression for the exponential attenuation coefficient experienced by an optical beam propagating through an ensemble of spherical scatterers. It is only necessary that the size distribution of the particles be describable in terms of a Poisson function. This description is, however, very general and has been used in discussions of atmospheric aerosols, ${ }^{9}$ clouds, ${ }^{10}$ and rain. ${ }^{11}$ A formula similar to Eq. (22) has been developed in an anomalous diffraction approach to determining the particle size distribution from its transparency. ${ }^{12}$ This approach has also been applied to various other distributions including the power-law, ${ }^{13}$ Maxwell distribution, ${ }^{14}$ and $\beta$-distribution, ${ }^{15}$ but these functions do not lead to a description in terms of elementary functions.

It is convenient to express Eq. (22) in terms of the normalized wavelength

$$
\lambda^{\prime}=\frac{\lambda}{4 \pi\left(\eta_{2}-\eta_{1}\right) a_{\max }}=\frac{1}{n_{\kappa}}
$$

with the result

$$
\begin{aligned}
& \frac{\gamma}{\pi a_{\max }^{2} N_{o}}=\frac{1}{n^{2}}\left\{2(n+2)(n+1)-\frac{4(n+1) n \lambda^{\prime}}{\left[1+\left(n \lambda^{\prime}\right)^{-2}\right]^{(n+2) / 2}}\right. \\
& \times \sin \left[(n+2) \tan ^{-1}\left(\frac{1}{n \lambda^{\prime}}\right)\right]+4\left(n \lambda^{\prime}\right)^{2}-\frac{4\left(n \lambda^{\prime}\right)^{2}}{\left[1+\left(n \lambda^{\prime}\right)^{-2}\right]^{(n+1) / 2}} \\
& \left.\times \cos \left[(n+1) \tan ^{-1}\left(\frac{1}{n \lambda^{\prime}}\right)\right]\right\} \cdot
\end{aligned}
$$

The general behavior of Eq. (24) is illustrated in Fig. 4, and the extinction coefficient for any Poisson-distributed ensemble of spherical scatterers can be read from this figure. These plots show the wavelength depen- dent attenuation that would be obtained for various size distributions of particles, and the distributions are also shown. It is apparent from the figure that increasing the relative width of the size distribution (decreasing $n$ ) increases the wavelength at which significant scattering occurs, provided that all other factors remain constant. This result confirms the reasonable fact that the long wavelength scattering is dominated by the largest particles. The figure also shows that the oscillations in the extinction are only significant for $n$ values greater than about $n \simeq 10$. In the limit of short wavelengths $\left(\lambda^{\prime} \rightarrow 0\right)$ all the curves approach the asymtotic value $2(n+2)(n+1) / n^{2}$.

\section{Absorbing Particles}

In previous applications of anomalous diffraction methods it has generally been assumed that the scattering particles introduce no absorption into the ray paths. The basic formulas are, however, equally valid when the index of refraction is complex, and the methods of the previous section can be extended to describe light extinction in ensembles of absorbing spheres. Our principal example of these results in-
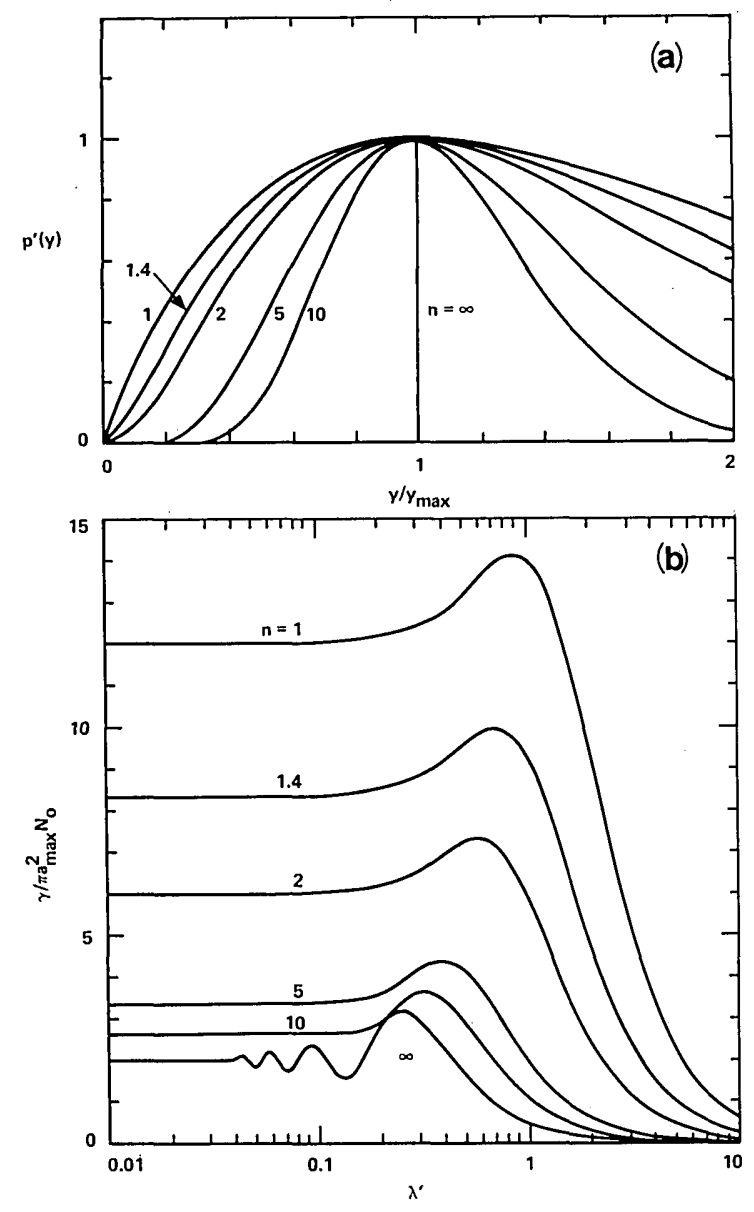

Fig. 4. Several normalized size distributions from Eq. (19) (a) together with the corresponding wavelength dependent extinction coefficient $\gamma / \pi a_{\max }^{2} N_{o}$ (b) for particles having a purely real index of refraction. 


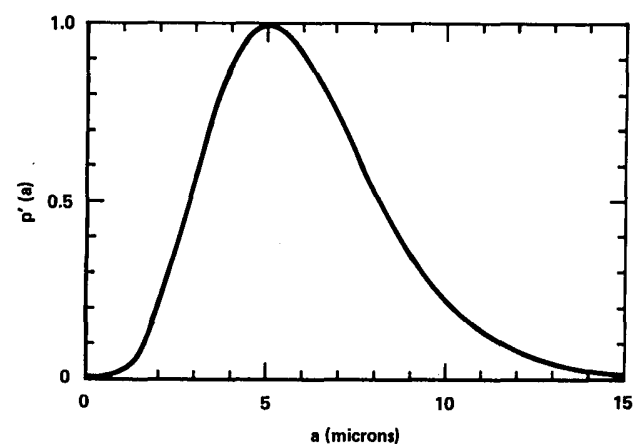

Fig. 5. Size distribution for a typical water fog with $a_{\max }=5 \mu \mathrm{m}$. The distribution parameter is $n=5$.

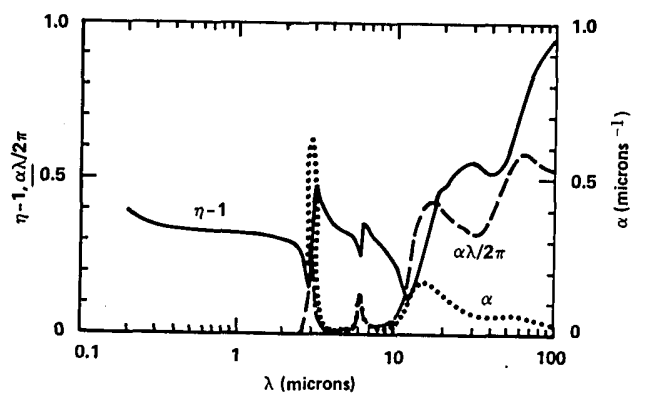

Fig. 6. Wavelength dependence of the relative index of refraction $\eta-1$ (solid line), imaginary index of refraction $\alpha \lambda / 2 \pi$ (dashed line), and amplitude absorption coefficient $\alpha$ (dotted line) based on Ref. 17.

volves the propagation of light beams through the distributions of water droplets that are characteristic of fog or rain. Absorption by the water droplets is of no consequence for visible light, but it can alter significantly the extinction coefficient for ir beams.

The relative extinction coefficient for an absorbing sphere can be derived from the anomalous diffraction theory, and the result is ${ }^{1}$

$$
\begin{aligned}
& Q_{\mathrm{ext}}=2-4 \exp (-\rho \tan \beta) \frac{\cos \beta}{\rho} \sin (\rho-\beta) \\
&-4 \exp (-\rho \tan \beta)\left(\frac{\cos \beta}{\rho}\right)^{2} \cos (\rho-2 \beta)+4\left(\frac{\cos \beta}{\rho}\right)^{2} \cos 2 \beta,
\end{aligned}
$$

where $\rho$ is defined as before in Eq. (17). The parameter $\beta$ can be related to the exponential amplitude absorption coefficients $\alpha_{1}$ outside of the sphere and $\alpha_{2}$ inside of the sphere by

$$
\tan \beta=\frac{\lambda\left(\alpha_{2}-\alpha_{1}\right)}{2 \pi\left(\eta_{2}-\eta_{1}\right)}=\lambda^{\prime}\left(\alpha_{2}-\alpha_{1}\right) 2 a_{\max }=\lambda^{\prime} \phi,
$$

where the factor $\phi$ is the attenuation exponent for central rays passing through the sphere in a nonabsorbing host medium $\left(\alpha_{1}=0\right)$. For nonabsorbing spheres $\beta$ and $\tan \beta$ vanish, and Eq. (25) reduces to Eq. (17).

When there is a distribution in the sizes of the scatterers, the over-all extinction coefficient is obtained from an appropriate integration. With the Poisson distribution of particle sizes this integration can be written

$$
\begin{aligned}
\gamma & =\frac{\pi a_{n}^{2} N_{o}}{\Gamma(n+1)} \int_{0}^{\infty}\left\{2-\frac{4 \cos \beta}{\kappa y} \exp (-\kappa y \tan \beta) \sin (\kappa y-\beta)\right. \\
& \left.+\frac{4 \cos ^{2} \beta}{\kappa^{2} y^{2}}[\cos 2 \beta-\exp (-\kappa y \tan \beta) \cos (\kappa y-2 \beta)]\right\} y^{n+2} \exp (-y) d y .
\end{aligned}
$$

The integrals are straightforward, and the result is

$$
\begin{gathered}
\gamma=\frac{\pi a_{\max }^{2} N_{o}}{n^{2}}\left\{2(n+2)(n+1)-\frac{4(n+1) \cos \beta}{\kappa\left[(1+\kappa \tan \beta)^{2}+\kappa^{2}\right]^{(n+2) / 2}}\right. \\
\times \sin \left[(n+2) \tan ^{-1}\left(\frac{\kappa}{1+\kappa \tan \beta}\right)-\beta\right]+\frac{4 \cos ^{2} \beta \cos 2 \beta}{\kappa^{2}} \\
-\frac{4 \cos ^{2} \beta}{\kappa^{2}\left[(1+\kappa \tan \beta)^{2}+\kappa^{2}\right]^{(n+1) / 2}} \\
\left.\times \cos \left[(n+1) \tan ^{-1}\left(\frac{\kappa}{1+\kappa \tan \beta}\right)-2 \beta\right]\right\} .
\end{gathered}
$$

It is convenient to express this equation in terms of the normalized wavelength $\lambda^{\prime}=(n \kappa)^{-1}$ and the absorption exponent $\phi$ yielding

$$
\begin{gathered}
\frac{\gamma}{\pi a_{\max }^{2} N_{o}}=\frac{1}{n^{2}}\left(2(n+2)(n+1)+\frac{4 n^{2} \lambda^{\prime 2}}{1+\lambda^{\prime 2} \phi^{2}}\left(\frac{2}{1+\lambda^{\prime 2} \phi^{2}}-1\right)\right. \\
-\frac{4 n(n+1) \lambda^{\prime}}{\left(1+\lambda^{\prime 2} \phi^{2}\right)^{1 / 2}}\left[\frac{n^{2} \lambda^{\prime 2}}{n^{2} \lambda^{\prime 2}(1+\phi / n)^{2}+1}\right]^{(n+2) / 2} \sin \{(n+2) \\
\left.\times \tan ^{-1}\left[\frac{1}{n \lambda^{\prime}(1+\phi / n)}\right]-\tan ^{-1}\left(\lambda^{\prime} \phi\right)\right\}-\frac{4 n^{2} \lambda^{\prime 2}}{1+\lambda^{\prime 2} \phi^{2}} \\
\times\left[\frac{n^{2} \lambda^{\prime 2}}{n^{2} \lambda^{\prime 2}(1+\phi / n)^{2}+1}\right]^{(n+1) / 2} \\
\left.\times \cos \left\{(n+1) \tan ^{-1}\left[\frac{1}{n \lambda^{\prime}(1+\phi / n)}\right]-2 \tan ^{-1}\left(\lambda^{\prime} \phi\right)\right\}\right) .
\end{gathered}
$$

As mentioned previously an important application of this formalism involves propagation through ensembles of water droplets, and we illustrate the results by considering propagation through fog. Droplet radii for fogs usually range between $1 \mu \mathrm{m}$ and $20 \mu \mathrm{m}$, and our assumed size distribution is shown in Fig. 5. The solid line is a renormalized plot of Eq. (19) with the index $n$ $=5$, and similar size distributions are typical for natural fog and clouds. ${ }^{16}$ In order to evaluate Eq. (29) it is also necessary to have data on the wavelength dependent index of refraction and absorption. Approximate plots of these quantities are given in Fig. 6 for wavelengths out to $100 \mu \mathrm{m} .{ }^{17}$

The wavelength dependent extinction coefficient of Eq. (29) is plotted in Fig. 7 for the fog described in Figs. 5 and 6 . The structure on this extinction curve can be identified with the detailed features of the refraction and absorption curves. The distinct minimum at about $11 \mu \mathrm{m}$, for example, is associated with the dip in the index of refraction at this wavelength. These results suggest that a laser at $11-\mu \mathrm{m}$ wavelength might be a near optimum choice for optical information transmission through fog. It is, however, important to recognize that the extinction coefficient derived here represents scattering and absorption by the water droplets only. In a realistic fog there is always a high level of humidity, and one must add on the appropriate extinction coefficient for propagation through the moist air. ${ }^{18}$ Water vapor and other atmospheric gases discriminate strongly against propagation of certain ir wavelengths. 
It should also be noted that the scattering of far ir wavelengths $(>30 \mu \mathrm{m})$ would probably be slightly different from the results shown in Fig. 7, because the anomalous diffraction method becomes less accurate for large values of the real or imaginary index of refraction.

In calculating extinction of light beams propagating through fog it is commonly assumed that absorption in the water droplets is negligible. Since the formalism of this section includes absorption, the validity of this assumption can be readily checked. The dashed line in Fig. 7 was derived taking full account of the size distribution and refraction implied by Figs. 5 and 6 , but the absorption was assumed to be negligible. Agreement between the two graphs is good out to a wavelength of about $5 \mu \mathrm{m}$, but at longer wavelengths substantial discrepancies occur. This result implies that absorption can have an important role in determining the ir transmission characteristics of fogs. It is also clear from this example that absorption by the droplets can lead to either an increase or a decrease in the over-all transmission.

Results for transmission through other size distributions of water droplets can be readily obtained from Eq. (29). For large water droplets the mathematics simplifies. As $a_{\max }$ increases, the value of $\lambda^{\prime}$ corresponding to a particular wavelength diminishes. In the limit of small $\lambda^{\prime}$ the right-hand side of Eq. (29) reduces to $2(n+2)(n+1) / n^{2}$ independent of the absorption factor $\phi$. Typical raindrops have sizes on the order of $1 \mathrm{~mm}$, so the extinction caused by the droplets would be constant over most of the visible and ir spectrum. On the other hand, droplet size distributions which are smaller and narrower than the one shown in Fig. 5 are also commonly observed in practice. For such fogs ir transmission would be better than that indicated in Fig. 7.

\section{Mass Density Normalization}

In most of the preceding analysis the extinction coefficient has been normalized in terms of the crosssectional area $\pi a_{\max }^{2}$ and the particle number density $N_{o}$.

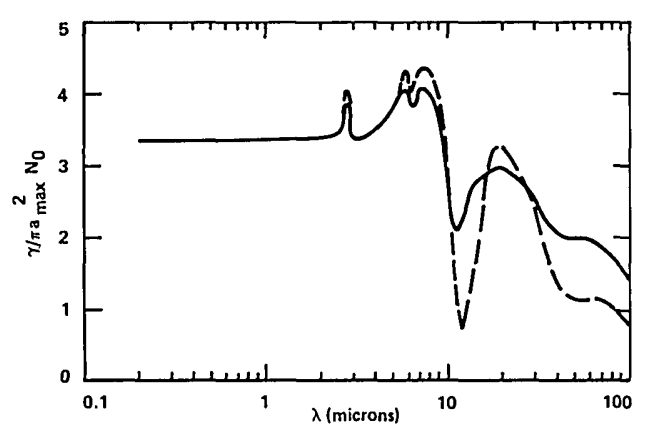

Fig. 7. Extinction coefficient as a function of wavelength for propagation through the typical water fog represented by Figs. 5 and 6 . It is assumed here that absorption in the surrounding water vapor is negligible. The dashed line shows the extinction coefficient that would be obtained if absorption in the droplets were neglected.

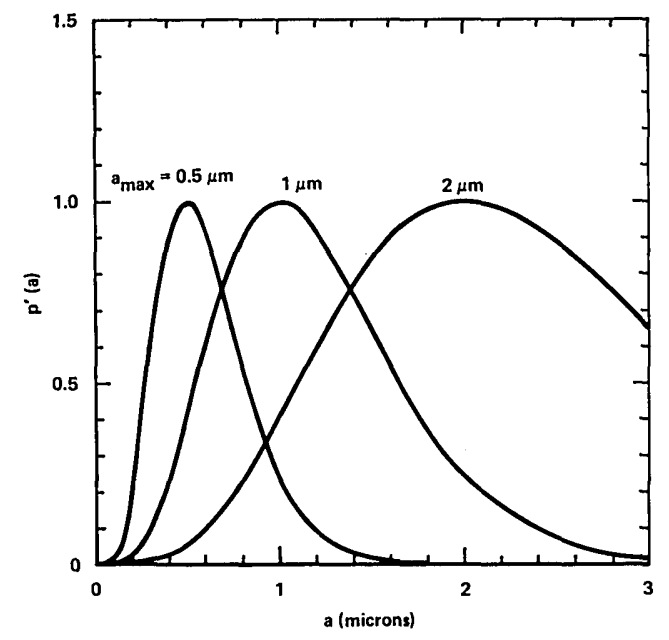

Fig. 8. Particle size distributions of three possible smoke ensembles with refractive index $\eta=1.5$, distribution parameter $n=4.6$, and maxima as labeled.

For some applications it is helpful to have available an alternative normalization in terms of the total mass density of the scattering particles. Such a normalization would be especially useful if, for example, one were analyzing the characteristics of an artificial smoke or fog and the total amount of suspendable material were specified. To obtain such a normalization it is first necessary to derive an explicit expression for the ensemble mass density $M$. In terms of the density of the individual particles $\mu$ the over-all mass density can be written

$$
M=\mu N_{o} \int_{0}^{\infty} \frac{4}{3} \pi a^{3} p(a) d a .
$$

With the particle size distribution of Eq. (19), Eq. (30) is

$$
M=\frac{\mu N_{o}}{\Gamma(n+1)} \frac{4}{3} \pi a_{n}{ }^{3} \int_{0}^{\infty}\left(\frac{a}{a_{n}}\right)^{n+3} \exp \left(-\frac{a}{a_{n}}\right) d\left(\frac{a}{a_{n}}\right) .
$$

This integral can be performed, and the result is

$$
\begin{gathered}
M=\mu N_{o} \frac{4}{3} \pi a_{n} 3 \frac{\Gamma(n+4)}{\Gamma(n+1)} \\
=\mu N_{o} \frac{4}{3} \pi a_{\max }^{3} \frac{(n+3)(n+2)(n+1)}{n^{3}} .
\end{gathered}
$$

With Eq. (32) it is straightforward to normalize any of the previous extinction formulas in terms of the mass density $M$ or the dimensionless mass ratio $r=M / \mu$. This parameter $r$ can also be expressed as the ratio of the volume of the scatterers to the total volume.

The significance of a mass density normalization can be best illustrated by means of a specific example. In Fig. 8 are three size distributions which correspond to plausible smoke ensembles. The index of refraction of the smoke particles is $\eta_{2}=1.5$, and the distribution parameter is $n=4.6$. These distributions have their maximum values at the radii $a_{\max }=0.5 \mu \mathrm{m}, 1 \mu \mathrm{m}$, and $2 \mu \mathrm{m}$. In Fig. 9 are the extinction coefficients for these smokes normalized to the mass ratio $r$. From these 


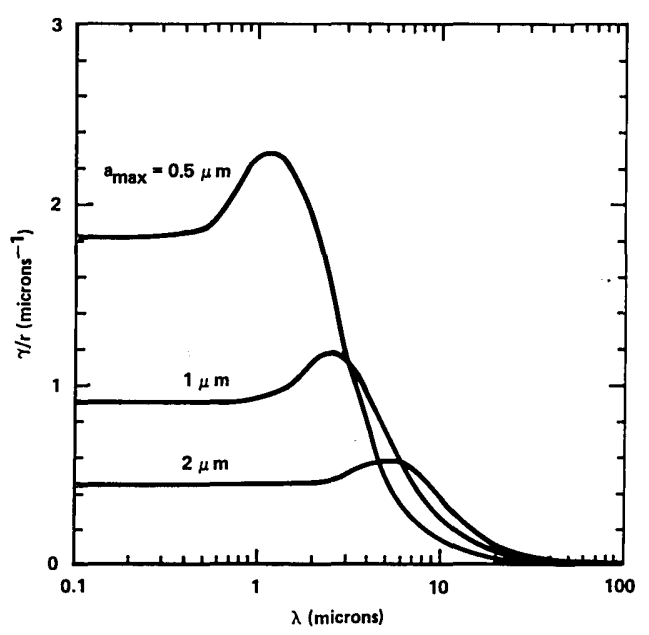

Fig. 9. Extinction coefficient as a function of wavelength for propagation through the smoke ensembles represented in Fig. 8 . The extinction is normalized in such a way that the total mass density is the same for the three ensembles.

plots it is clear that an increase in particle size leads to an increase in the extinction at a long wavelength, such as $10 \mu \mathrm{m}$. At the same time this size increase yields a more substantial reduction in the extinction at the shorter wavelengths. These results imply that the long wavelengths have an advantage for propagation in environments where a limited total mass of scattering material is anticipated. It also follows from Fig. 9 that a really optimum system might include a laser in the short wavelength or visible region of the spectrum as well as an ir laser source.

\section{Conclusion}

Light extinction due to scattering from suspended particles is an important consideration in various optical communication and optical radar applications. It has been shown here that the anomalous diffraction method makes it possible to develop explicit analytic formulas governing the extinction, provided that the size distribution can be described in terms of certain Poissonlike distribution functions. Data fitting techniques for use with these functions have also been described. These methods could be generalized to more complicated particle ensembles by fitting the size characteristics to a sum of Poisson functions. Most previous discussions of extinction have been limited to particles having a real index of refraction. The analysis here has included particles with arbitrary complex dielectric properties, and the significance of particle absorption has been illustrated in a discussion of beam propagation through fog.

The author also holds an appointment at Northrop Research \& Technology Center. This work was sponsored by Northrop and supported in part by the NSF.

\section{References}

1. H. C. van de Hulst, Light Scattering by Small Particles (Wiley, New York, 1957), Chap. 11.

2. M. Nakagaki and T. Shimoyama, Bull. Chem. Soc. Jpn. 37, 1634 (1964).

3. M. Kerker, The Scattering of Light and Other Electromagnetic Radiation (Academic, New York, 1969), p. 357.

4. A. Hald, Statistical Theory with Engineering Applications (Wiley, New York, 1962).

5. W. F. Espenscheid, M. Kerker, and E. Matijević, J. Phys. Chem. 68, 3093 (1964).

6. M. Abramowitz and I. A. Stegun, Eds., Handbook of Mathematical Functions, NBS (U.S.) Appl. Math. Ser. 55 (U.S. GPO, Washington, D.C., 1964), p. 18.

7. R. Penndorf, J. Phys. Chem. 62, 1537 (1958).

8. I. S. Gradshteyn and I. W. Ryzhik, Table of Integrals Series and Products (Academic, New York, 1965), pp. 3.944-5,6.

9. J. Rocard, Rev. Opt. 9, 97 (1930).

10. A. Kh. Khrgian and I. P. Mazin, Tr. Tsentr. Aerolog. Obs. No. 7 (1952).

11. E. A. Polyakova and K. S. Shifrin, Tr. Gl. Geofiz. Obs. No. 109 (1961).

12. K. S. Shifrin, A. Ya. Perelman, and V. G. Bakhtiyarov, Opt. Spectrosc. 20, 386 (1966) and references.

13. A. Ya. Perelman and K. S. Shifrin, Opt. Spectrosc. 26, 548 (1969).

14. G. Hilbig, Optik 23, 313 (1966).

15. A. Ya. Perelman and K. S. Shifrin, Opt. Spectrosc. 27, 66 (1969).

16. See, for example, R. T. Ryan, H. H. Blau, Jr., P. C. von Thuna, and M. L. Cohen, J. Appl. Meteorol. 11, 149 (1972).

17. G. M. Hale and M. R. Querry, Appl. Opt. 12, 555 (1973).

18. W. L. Wolfe, Ed., Handbook of Military Infrared Technology (U.S. GPO, Washington, D.C., 1965), Chap. 6.

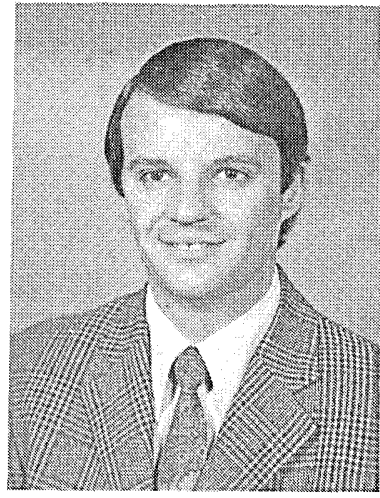

Peter C. Schultz of Corning Glass Works, recipient of the first Woldemar A. Weyl International Glass Science Award, sponsored by Penn State University 